\title{
ANÁLISES QUALI E QUANTITATIVAS DE MICOTOXINAS EM ÁGUAS DA CADEIA PRODUTIVA DO ARROZ POR CCD E CCDAE
}

\author{
Clarice Caldeira Leite, Jaqueline Garda-Buffon*, Carlos Alberto Fagundes e Eliana Badiale-Furlong
}

Universidade Federal do Rio Grande, CP 474, 96201-900 Rio Grande - RS, Brasil

Recebido em 20/2/12; aceito em 4/7/12; publicado na web em 17/9/12

\begin{abstract}
QUALITATIVE AND QUANTITATIVE MYCOTOXIN ANALYSES IN RESIDUAL WATER OF RICE PRODUCTION CHAIN BY TLC AND HPTLC. This study validated a simple and applied method for determining mycotoxins aflatoxin $\mathrm{B}_{1}$, aflatoxin $\mathrm{B}_{2}$, ochratoxin A, zearalenone and deoxynivalenol, in water from the rice production chain. Five solvent combinations for extraction were tested, with quantification performed by TLC/HPTLC and confirmation by LC-MS/MS. Mycotoxins in water from field and rice industries were evaluated. Mycotoxin recovery levels were around $90 \%$. Two samples from rice parboiling waste were contaminated (deoxynivalenol/aflatoxin $\mathrm{B}_{1}, 110 / 9 \mathrm{ng} \mathrm{mL}^{-1}$; and deoxynivalenol, $100 \mathrm{ng} \mathrm{mL}^{-1}$ ). Zearalenone, deoxynivalenol and ochratoxin $\mathrm{A}$ (36, 30 and $28 \%$ ) were carried to soaking water during parboiling.
\end{abstract}

Keywords: method validation; mycotoxins; water.

\section{INTRODUÇÃO}

O cultivo de arroz, em diferentes regiões do mundo, é realizado sob irrigação artificial, como acontece no Rio Grande do Sul, o maior produtor nacional do grão. O sistema consiste em recobrir o campo com água de mananciais hídricos até uma determinada etapa de desenvolvimento da cultura. ${ }^{1,2}$ Durante o beneficiamento do grão, a água é empregada para limpeza de instalações/equipamentos e para o processo de parboilização, onde é empregada em uma relação de 1:4 em massa:volume de grão e água, requerida para encharcamento do grão realizado previamente ao beneficiamento. ${ }^{1-3}$

No arroz os contaminantes microbianos ou químicos, quando presentes, encontram-se principalmente nas camadas mais externas do grão. ${ }^{4-6}$ A migração destes para água durante a irrigação é bastante provável, com consequente distribuição pelos mananciais, ou na etapa de industrialização, comprometendo a qualidade do efluente a ser descartado. Embora diversos solutos possam comprometer a qualidade do meio ambiente, as micotoxinas chamam a atenção por sua resistência a tratamentos físicos e químicos mesmo os drásticos, ${ }^{7-9}$ o que sugere a presença em quantidades residuais que, difundidas pelo ambiente hídrico, podem atingir animais e humanos usuários do recurso. ${ }^{10,11}$

A ingestão de alimentos ou água contaminados com estes compostos pode causar efeitos nocivos à saúde humana e animal, como depressão do sistema imunológico e neurológico, distúrbios gastrointestinais, renais e até câncer. ${ }^{6-9}$

Muitos estudos vêm sendo conduzidos para avaliar a presença de micotoxinas em diferentes matérias-primas e alimentos. ${ }^{3,5,8,11,12,14,15}$ Estes estudos motivaram, em 2011, a implantação de legislação mais abrangente e rígida no Brasil, ${ }^{13}$ em função da ocorrência destes compostos. No entanto, o impacto destes contaminantes no ambiente hídrico ainda é pouco estudado, requerendo uma avaliação de risco, especialmente em regiões produtoras de arroz irrigado, como o Rio Grande do Sul, onde abundam recursos hídricos para o cultivo irrigado do arroz, ${ }^{1,2}$ outras culturas e para consumo humano e animal. Nesta região também se encontram as maiores empresas produtoras do país, especialmente do arroz parboilizado. ${ }^{1,2}$

Outro fato que justifica a preocupação com a contaminação de águas com micotoxinas são os frequentes relatos de

*e-mail: jaquelinebuffon@ furg.br ocorrência de fungos toxigênicos e micotoxinas em arroz e seus

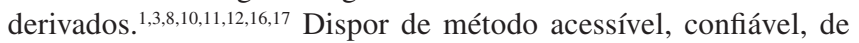
baixo custo, que gere pouco resíduo para descarte, e apresente ampla aplicabilidade para iniciar a avaliação do risco de danos ao ambiente hídrico decorrente desta cadeia produtiva é uma estratégia primordial para preservação dos recursos hídricos.

Neste sentido, a técnica de cromatografia em camada delgada empregando cromatofolhas recobertas com sílica com menor espessura $(5 \mu \mathrm{m})$ e nanopartículas de sílica apresenta as vantagens da simplicidade de execução, rapidez, menor geração de resíduo, possibilidade de determinação simultânea de várias amostras com eficiência resultante da uniformidade da fase estacionária, reutilização do eluente e baixo custo de manutenção. ${ }^{18,19}$ Estas características a torna acessível a laboratórios de rotina simples próximos aos locais de cultivo, em plantas industriais ou estações de tratamento de efluente.

No caso da detecção de analitos fluorescentes, como são algumas micotoxinas, os limites de detecção atingem a ordem de $1 \mathrm{ng}$ e a linearidade pode ser visualizada em concentrações até 15 vezes mais elevadas..$^{18,20-22}$ Estes níveis de sensibilidade são adequados para atender às recomendações de diversos órgãos de normalização analítica, ambientais e de segurança alimentar. ${ }^{12,13,23-25}$ Estes aspectos nortearam este trabalho cujo objetivo foi adaptar e validar um método multimicotoxinas simples e aplicável para determinar aflatoxina $\mathrm{B}_{1}$ $\left(\right.$ AFA $\left.B_{1}\right)$, aflatoxina $B_{2}\left(A F A B_{2}\right)$, ocratoxina $A(O T A)$, zearalenona (ZEA) e deoxinivalenol (DON) em águas da cadeia produtiva do arroz, visando disponibilizar um procedimento exequível que possa contribuir para a tomada de medidas preventivas contra danos ambientais decorrentes de cultivo e industrialização de grãos, tendo o arroz como ponto de partida.

\section{PARTE EXPERIMENTAL}

\section{Solventes e reagentes}

Os solventes metanol, acetonitrila, acetato de etila, clorofórmio e outros reagentes de uso geral adquiridos foram de grau analítico das marcas Vetec (Brasil) e Merck (Alemanha). Os padrões das micotoxinas AFA $\mathrm{B}_{1}$, AFA $\mathrm{B}_{2}$, OTA, ZEA e DON foram adquiridos da Sigma Chemical Co. (USA) na forma de cristais. 
As placas de cromatografia de camada delgada (CCD) empregadas foram de sílica gel G 60, com 0,25 mm de espessura da marca Merck (Brasil) e placas de cromatografia de camada delgada de alta eficiência (CCDAE) de nanossílica gel de 0,2 mm marca Alugram (Alemanha).

\section{Soluções padrão de micotoxinas}

As soluções padrão de micotoxinas utilizadas foram preparadas conforme método descrito por Bennett e Shotwell. ${ }^{3,10,11,12,16}$ Este consiste em se dissolver $5 \mathrm{mg}$ de cada padrão em $100 \mathrm{~mL}$ da mistura de benzeno-acetonitrila (95:5). As soluções estoques foram diluídas de modo a resultar em soluções de trabalho cujas concentrações foram confirmadas espectrofotometricamente, em equipamento da marca Varian/Cary - 100 Conc. As concentrações das soluções padrões foram estimadas a partir da absorvância no comprimento de onda e absortividade molar específica de cada micotoxina. ${ }^{20}$ Foram empregadas as soluções de trabalho com as seguintes concentrações 1,$25 ; 1,5 ; 7,4 ; 25,6$ e $36,5 \mu \mathrm{gL}^{-1}$ para AFA B AFA B $_{2}$, OTA ZEA e $\mathrm{DON}$, respectivamente, que foram acondicionadas em frascos âmbar vedados e armazenados a $-18{ }^{\circ} \mathrm{C}$ até o momento do uso.

\section{Condições cromatográficas para determinação de micotoxinas}

Foram utilizadas placas $\mathrm{CCD}$ previamente ativadas a $105^{\circ} \mathrm{C}$ por 15 min e placas de CCDAE sem prévia ativação. As soluções padrões foram aplicadas nas placas com microsseringas, marca Hamilton, em volumes variando entre 5 e $30 \mu \mathrm{L}$, abrangendo faixa de linearidade de visualização da fluorescência dos padrões sob luz UV. As micotoxinas foram eluídas com uma mistura dos solventes tolueno, acetato de etila e ácido fórmico na proporção de 60:40:10 (v/v/v)..$^{3,14,17,18}$ Após a eluição, as placas foram secas a $25^{\circ} \mathrm{C}$ e as AFAS e OTA visualizadas diretamente em câmara sob UV (366 e 254 nm), DON e ZEA foram visualizadas após derivação, que consistia em mergulhar as placas em solução etanólica de cloreto de alumínio $15 \%$ (m/v), seguindo-se aquecimento a $130{ }^{\circ} \mathrm{C}$ por $10 \mathrm{~min} .{ }^{21}$ Foram determinados os fatores de retardamento (Rf), limites de detecção (LD) e a linearidade de detecção para os dois tipos de fase estacionária. O LD foi determinado aplicando-se nas placas soluções padrão que foram sendo diluídas até a detecção do ponto de menor fluorescência visualizado por três analistas treinados. A massa de micotoxina detectável foi estimada a partir deste volume de padrão aplicado na placa e o limite de quantificação definido como três vezes superior à massa do LD. A linearidade da detecção da fluorescência das micotoxinas foi determinada aplicando-se na placa soluções padrão em concentrações crescentes, sendo considerado o limite máximo o ponto cuja fluorescência podia ser distinguida do anterior por três analistas treinados. ${ }^{18,20,23}$

\section{Estabelecimento das condições para a extração simultânea das micotoxinas}

Foram testadas cinco condições de partição para extração simultânea de AFA B ${ }_{1}$, AFA B ${ }_{2}$, OTA, ZEA e DON empregando $25 \mathrm{~mL}$ de água residuária de parboilização de arroz artificialmente contaminada com padrões de micotoxinas. As concentrações foram estabelecidas a partir da definição dos LD em placas CCD e CCDAE, testados em três níveis de fortificação especificados na Tabela 1. Os experimentos para estabelecimento das condições de extração foram realizados em triplicata.

A contaminação artificial das amostras de água foi realizada adicionando-se volumes de soluções padrão em erlenmeier de 50 $\mathrm{mL}$, de onde os solventes foram evaporados sob atmosfera de nitrogênio. Em seguida, foram adicionados $25 \mathrm{~mL}$ de água residuária do
Tabela 1. Níveis de fortificação empregados para padronização da extração das micotoxinas da amostra de água de parboilização

\begin{tabular}{cccc}
\hline Micotoxina & $\begin{array}{c}\text { Nível 1 } \\
\left(\mathrm{ng} \mathrm{mL}^{-1}\right)\end{array}$ & $\begin{array}{c}\text { Nível 2 } \\
\left(\mathrm{ng} \mathrm{mL}^{-1}\right)\end{array}$ & $\begin{array}{c}\text { Nível 3 } \\
\left(\mathrm{ng} \mathrm{mL}^{-1}\right)\end{array}$ \\
\hline AFA B $_{1}$ & 9 & 18 & 27 \\
AFA B $_{2}$ & 6 & 12 & 18 \\
OTA & 15 & 30 & 60 \\
ZEA & 70 & 140 & 210 \\
DON & 65 & 130 & 200 \\
\hline
\end{tabular}

encharcamento do arroz, submetidos à agitação orbital a $150 \mathrm{rpm}$ a $30{ }^{\circ} \mathrm{C}$ por 30 min antes da extração líquido-líquido testada em cada experimento.

O experimento 1 consistiu em homogeneizar em blender $25 \mathrm{~mL}$ de amostra contaminada, $130 \mathrm{~mL}$ de metanol e $15 \mathrm{~mL}$ de solução de cloreto de potássio $5 \%$ durante $5 \mathrm{~min}$. A mistura foi clarificada com $75 \mathrm{~mL}$ de sulfato de amônio $30 \%(\mathrm{~m} / \mathrm{v})$ e $25 \mathrm{~mL}$ de celite. $\mathrm{O}$ filtrado foi submetido a três partições sucessivas com $10 \mathrm{~mL}$ de

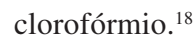

No experimento 2, foram homogeneizados em blender $25 \mathrm{~mL}$ de água de parboilização contaminada e $150 \mathrm{~mL}$ de uma mistura de acetonitrila e água destilada e deionizada na proporção de 14:1 durante $5 \mathrm{~min}$, seguido de três partições com $10 \mathrm{~mL}$ de clorofórmio. ${ }^{26}$ No experimento 3, foram homogeneizados em blender $25 \mathrm{~mL}$ de amostra e $120 \mathrm{~mL}$ de uma mistura de metanol e acetato de etila na proporção de 9:3 durante 5 min, seguido de três partições sucessivas com $10 \mathrm{~mL}$ de clorofórmio. ${ }^{27-29}$ A mistura extratora do experimento 4 foi constituída por metanol, acetato de etila na proporção de 8:2 homogeneizados em blender durante $5 \mathrm{~min}$, seguido de três partições com $10 \mathrm{~mL}$ de clorofórmio. ${ }^{16} \mathrm{O}$ experimento 5 consistiu em se realizar três extrações sucessivas de $25 \mathrm{~mL}$ de amostra com $10 \mathrm{~mL}$ de clorofórmio. ${ }^{16}$

Ao final, os extratos clorofórmicos correspondentes a cada experimento foram coletados em frascos âmbar, secos sob atmosfera de nitrogênio a $25^{\circ} \mathrm{C}$ e vedados com tampa de rosca.

Os resíduos secos foram ressuspensos em $200 \mu \mathrm{L}$ de benzeno, agitados em banho ultrassom por $60 \mathrm{~s}$ e 20 ou $30 \mu \mathrm{L}$ aplicados em CCD e CCDAE para determinação das micotoxinas. A identificação das micotoxinas foi realizada por comparação com os Rf dos padrões e a quantificação foi realizada comparando-se a intensidade da fluorescência com série de padrões aplicados às placas em massas crescentes para cada micotoxina.

$\mathrm{O}$ experimento que resultou em maiores percentuais de recuperação para todas as micotoxinas foi repetido usando-se amostras contaminadas em três níveis para a confirmação dos resultados empregando cromatografia líquida acoplada a espectrômetro de massas em sequencial, no Laboratório de Contaminantes Orgânicos e Metálicos da Escola de Química e Alimentos da FURG.

O equipamento utilizado foi um cromatógrafo líquido Alliance Separtaions Module 2695, da marca Waters (Milford, USA), equipado com: amostrador automático; bomba quaternária; forno para coluna e sistema de desgaseificação; detector de massas (Micromass Quatro Micro API) com fonte API, utilizando eletrospray, atuando no modo SRM; sistema de aquisição de dados software MassLynx e QuanLynx 4.1; sistema gerador de nitrogênio Peak Scientifics e coluna XTerra ${ }^{\circledR}$ MS C18 (50 x 3 mm x 3,5 $\mu \mathrm{m}$ diâmetro interno).

A fase móvel empregada foi composta por uma mistura de metanol e solução de ácido acético a 0,5\% (90:10 v/v), numa vazão de $0,2 \mathrm{~mL} \mathrm{~min}^{-1}$ e volume de injeção $10 \mu \mathrm{L}$. No detector de massas, as condições foram voltagem capilar $3,5 \mathrm{kV}$, temperatura da fonte 
Tabela 2. Resultados dos parâmetros otimizados dos compostos analisados por LC- MS/MS

\begin{tabular}{ccccc}
\hline Micotoxinas & Transição $(\mathrm{m} / z)$ & $\mathrm{t}_{\mathrm{R}}(\mathrm{min})$ & Equação da reta & Coeficiente de correlação \\
\hline Aflatoxina $\mathrm{B}_{1}$ & $313>241^{b} 313>285$ & 1,58 & $\mathrm{y}=254550 \mathrm{x}+186,024$ & 0,9947 \\
Aflatoxina $\mathrm{B}_{2}$ & $315>287^{b} 315>259$ & 1,65 & $\mathrm{y}=145703 \mathrm{x}+170,848$ & 0,9969 \\
Ocratoxina A & $404>239^{b} 404>358$ & 1,80 & $\mathrm{y}=109432 \mathrm{x}+256,753$ & 0,9874 \\
Zearalenona $^{a}$ & $317>131^{b} 317>175$ & 1,69 & $\mathrm{y}=5294,25 \mathrm{x}+3,14475$ & 0,9965 \\
Deoxinivalenol $^{a}$ & $355>58,8355>295,2$ & 1,47 & $\mathrm{y}=241,819 \mathrm{x}+1,93418$ & 0,9991 \\
\hline
\end{tabular}

${ }^{a}$ Fonte de ionização em modo negativo; ${ }^{b}$ transição usada para quantificação.

$120{ }^{\circ} \mathrm{C}$ e dessolvatação $400^{\circ} \mathrm{C}$, determinadas no modo positivo para AFA $B_{1}$, AFA $B_{2}$, OTA, e negativo para DON e ZEA (Tabela 2). ${ }^{30}$

\section{Indicativos de mérito do método}

Escolhido o procedimento de extração e as condições cromatográficas ideais, o método foi avaliado quanto aos valores dos indicativos de eficiência para aplicabilidade em água, sendo: limite de detecção do método (LD) expresso em ng $\mathrm{mL}^{-1}$; limite de quantificação (LQ) expresso em ng $\mathrm{mL}^{-1}$; exatidão através dos ensaios de recuperação e precisão, pelo cálculo dos coeficientes de variação de 6 análises consecutivas. ${ }^{23-26}$

\section{Aplicabilidade}

O método proposto foi empregado para realizar um levantamento de ocorrência das micotoxinas em amostras coletadas em campos experimentais, realizadas em triplica, em diferentes períodos da irrigação (16). Também foram coletadas amostras de água residuária da etapa de encharcamento (6) durante o processo de parboilização de arroz em empresas beneficiadoras da região sul do Brasil.

Amostras de água residuária de encharcamento foram produzidas em experimentos de laboratório, contaminando-se artificialmente arroz em casca. Estas alíquotas, $60 \mathrm{~g}$ de arroz em casca isento de contaminação com micotoxinas, foram acomodadas em recipientes de vidro (volume 0,5 L) e contaminadas artificialmente com solução padrão contendo mistura de concentração conhecida das micotoxinas. Esta solução foi distribuída em diferentes pontos da superfície da massa da amostra, evitando-se a aderência nas paredes dos recipientes, que foram deixados em temperatura ambiente $\left(25^{\circ} \mathrm{C}\right)$.

As massas de micotoxinas empregadas para contaminação do arroz em casca, dois níveis, estão apresentadas na Tabela 3. Os níveis adotados foram estimados a partir de trabalhos anteriores que avaliaram a migração de micotoxinas para o interior do grão durante o processo de parboilização, além dos LD. ${ }^{10,14}$

Tabela 3. Contaminação artificial do arroz em casca para a parboilização em laboratório

\begin{tabular}{ccc}
\hline Micotoxinas & Nível $1\left(\mu \mathrm{g} \mathrm{g}^{-1}\right)$ & Nível $2\left(\mu \mathrm{g} \mathrm{g}^{-1}\right)$ \\
\hline AFA B $_{1}$ & 0,2 & 0,4 \\
OTA & 2,5 & 5,0 \\
ZEA & 10,0 & 20,0 \\
DON & 12,0 & 16,0 \\
\hline
\end{tabular}

As amostras contaminadas e controle foram submetidas a encharcamento com $180 \mathrm{~mL}$ de água destilada em intervalos de tempos pré-estabelecidos de 4 e $6 \mathrm{~h}$ a $60^{\circ} \mathrm{C}$. Os níveis adotados foram estimados a partir dos valores de $\mathrm{LD}$ e de trabalhos anteriores que avaliaram a migração de micotoxinas para o interior do grão durante o processo de parboilização. ${ }^{10,14}$ As amostras de águas provenientes da drenagem dos diferentes recipientes de encharcamento foram analisadas quanto à presença das micotoxinas pelo método adaptado.

\section{RESULTADOS E DISCUSSÃO}

\section{Condições cromatográficas para determinação das micotoxinas}

A CCD e, atualmente, a CCDAE vêm sendo recomendadas para detecção de compostos fluorescentes em matrizes pouco complexas, ${ }^{18,24}$ mesmo com a disponibilidade de instrumentação analítica dotada de detector em fluxo ou de imunofluorescência. Especialmente por ser a técnica adaptável para determinações precisas em condições de infraestrutura simples, com vantagem sobre as técnicas de imunoafinidade, por propiciar a avaliação de multitoxinas em diversas amostras simultaneamente.

Neste trabalho, onde as amostras foram águas provenientes de diferentes etapas da cadeia produtiva do arroz, a utilização de CCD e CCDAE foi considerada promissora para compor um método exequível, sem a necessidade de infraestrutura sofisticada, resultando em um procedimento rápido para controle em rotina de produção e com pequena geração de resíduos para descarte.

Os valores de Rf, LD e LQ das micotoxinas avaliadas em CCD e CCDAE estão descritos na Tabela 4. A estimativa da resolução cromatográfica apresentou valores entre 0,2 e 0,8 nos dois sistemas cromatográficos, mostrando a adequação da separação obtida nas condições de eluição adotadas. Da mesma forma, os valores de LD e linearidade foram aplicáveis ao acompanhamento da etapa de preparação de amostras de água, pois atendem às recomendações da literatura para micotoxinas em outras matrizes, ${ }^{24,25}$ visto que não estão disponíveis limites legais para amostras de água. Cabe salientar que o emprego de placas CCDAE diminuiu em até três vezes os valores dos LD das micotoxinas AFA $\mathrm{B}_{2}$, ZEA e DON.

Tabela 4. Características das técnicas cromatográficas para a determinação de micotoxinas

\begin{tabular}{ccccccc}
\hline \multirow{2}{*}{ Micotoxina } & Rf & \multicolumn{3}{c}{ LD ng } & \multicolumn{3}{c}{ LQ ng } \\
\cline { 2 - 7 } & CCD & CCDAE & CCCD & CCDAE & CCD & CCDAE \\
\hline AFA B1 & 0,17 & 0,64 & 1,5 & 1,0 & 4,5 & 3 \\
AFA B2 & 0,29 & 0,68 & 5 & 1,2 & 15 & 4 \\
OTA & 0,66 & 0,80 & 12 & 7,0 & 36 & 21 \\
ZEA & 0,71 & 0,90 & 76 & 26 & 228 & 78 \\
DON & 0,13 & 0,48 & 73 & 27 & 219 & 71 \\
\hline
\end{tabular}

CCD: cromatografia de camada delgada; CCDAE: cromatografia de camada delgada de alta eficiência; Rf: fator de retardamento; LD: limite de deteç̧ão; LQ: limite de quantificação

Em estudo realizado por Garda et al. ${ }^{16}$ para determinação de micotoxinas em cerveja por CCD, foram obtidos resultados semelhantes 
para AFAs 4,0 ng, ZEA 30 ng, OTA 7 ng e DON 30 ng.

\section{Extração das micotoxinas}

Desde 1976 a extração em fase sólida tem sido adotada para preparo de amostras para análises cromatográficas posteriores, ${ }^{31}$ pelo menor consumo de solventes, não formação de emulsões, eficiência de recuperação, entre outras vantagens. No entanto, neste trabalho a proposta foi adaptar um procedimento acessível e rápido para verificar a ocorrência de micotoxinas de famílias químicas distintas, que demandariam a necessidade de emprego de mais de um tipo de sorvente para cada amostra, o que acarretaria em custo e aumento do tempo de execução do procedimento. Além disso, os sorventes, embora disponíveis para diferentes aplicações, dificilmente são reutilizáveis e, portanto, geram material para descarte sólido. ${ }^{32}$

A extração líquido-líquido foi adotada, pois tem sido relatada na literatura como técnica tradicionalmente empregada para matrizes líquidas para determinação de multimicotoxinas. ${ }^{16,18,21,27-29}$ Os solventes estudados também vêm sendo mencionados na literatura para extração destes analitos em grãos e bebidas fermentadas com sucesso, apesar da complexidade das matrizes. . $10,16,17,18,21,22,26$ Foram necessárias adaptações para adequar as condições de interação dos solventes mencionados pela literatura ao estado físico da matriz, considerando-se a proporção entre extratores, volume da amostra e tipo de interferentes.

A amostra empregada para o estabelecimento das condições de extração foi a água residuária da etapa de encharcamento durante a parboilização do arroz em casca isento de contaminação. Esta escolha foi por representar a condição de interferência com maior efeito durante o monitoramento de águas da cadeia produtiva do arroz. ${ }^{15}$

A Tabela 5 apresenta os resultados expressos como porcentagem de recuperação e coeficiente de variação dos testes de extração. Os níveis de recuperação das micotoxinas obtidos no experimento 1 mostraram que a mistura extratora foi ineficiente, devido a sua grande variabilidade e por não recuperar as toxinas de Fusarium (DON e ZEA) dentro dos limites de detecção das técnicas cromatográficas adotadas. Recuperar estas toxinas é importante, especialmente quando se considera que espécies típicas de Fusarium podem infectar o arroz no campo, especialmente tendo como meta monitorar também a contaminação das águas de irrigação, nas quais representariam o maior risco..$^{5,8-10,26,27}$

No experimento 4 não foi possível recuperar nenhuma micotoxina de interesse, ao passo que no experimento 5, onde foi realizada a partição diretamente com o clorofórmio, os níveis de recuperação variaram entre 72 a 97\%, conforme recomendações do INMETRO, ${ }^{25}$ Comunidade Europeia ${ }^{24}$ e outros órgãos que dispõem sobre indicativos de confiabilidade analítica. ${ }^{23}$ Garda et al. ${ }^{16}$ encontraram, empregando este mesmo solvente para amostras de cerveja, níveis de recuperação similares. O clorofórmio mostrou-se adequado à extração simultânea das micotoxinas também pela repetibilidade, demonstrada por coeficientes de variação inferiores a $20 \% .{ }^{23,25}$

$\mathrm{O}$ experimento 3 também foi promissor, pois $\mathrm{ZEA} \mathrm{e} \mathrm{AFAB}_{2}$ foram recuperadas em níveis próximos a $70 \%$ da contaminação inicial. No experimento 2, apenas a ZEA foi recuperada em níveis aceitáveis com boa precisão. ${ }^{23,25}$

A condição de partição que resultou em maior recuperação das micotoxinas simultaneamente teve seus resultados confirmados por LC/MS-MS e a comparação dos resultados mostrou um coeficiente de correlação de 0,87 para três níveis de contaminação testados. Isto demonstra que a extração líquido-líquido para determinação simultânea das micotoxinas de águas provenientes da cadeia produtiva do arroz é confiável para aplicação em rotina rápida na avaliação de qualidade de água ou efluente da indústria. Outra vantagem foi que mesmo não empregando a extração em fase sólida, o volume de solvente utilizado foi comparável ao desse, sem resultar em material para descarte sólido.

\section{Método adaptado e validado}

A partir destas considerações, o método proposto para extração de multimicotoxinas consistiu em tomar $25 \mathrm{~mL}$ da amostra e submeter a três extrações líquido-líquido sucessivas de $10 \mathrm{~mL}$ de clorofórmio. Homogeneizar as três alíquotas eluídas em banho ultrassom e separar em três porções, sendo duas de $10 \mathrm{~mL}$ e uma com o restante. Secar os extratos em banho-maria a $50{ }^{\circ} \mathrm{C}$ sob atmosfera de nitrogênio. Para a etapa cromatográfica, ressuspender o resíduo seco em $200 \mu \mathrm{L}$ de benzeno, homogeneizar em banho ultrassom e aplicar 20 e $30 \mu \mathrm{L}$ em placas CCD e CCDAE, respectivamente.

Os indicativos de mérito do método estão apresentados na Tabela 6. As faixas de linearidade abrangidas pelo método completo foram estimadas considerando-se as etapas de extração e determinação cromatográfica. As variações foram entre 0,9 e $13,2 \mathrm{ng} \mathrm{mL}^{-1}$ para AFA $B_{1} ; 0,72$ e 4,2 $\mathrm{ng} \mathrm{mL}^{-1}$ para AFA $\mathrm{B}_{2} ; 4,2$ e 40,8 $\mathrm{ng} \mathrm{mL}^{-1}$ para OTA; 15,6 e $230 \mathrm{ng} \mathrm{mL}^{-1}$ para ZEA e, 16,2 e $219 \mathrm{ng} \mathrm{mL}^{-1}$ para DON. Essas faixas amplas permitem sua aplicação em amostras com diferentes níveis de contaminação, como pode ocorrer em locais de cultivo ou beneficiamento.

\section{Ocorrência de micotoxinas em águas coletadas da cadeia produtiva do arroz}

O método validado aplicado para avaliar a ocorrência de micotoxinas em 22 amostras da água da cadeia produtiva do arroz mostrou que não havia contaminação detectável em águas de irrigação, dentro dos LD e LQ obtidos nas condições do método. No entanto, duas amostras de água residuária do processo de parboilização estavam contaminadas, uma com AFA $B_{1}$ e DON, respectivamente, com 9 e $110 \mathrm{ng} \mathrm{mL}^{-1}$ e outra com DON, $100 \mathrm{ng} \mathrm{mL}^{-1}$ (Figura 1).

Esses resultados sugerem que os grãos submetidos ao processo industrial estavam contaminados e que as micotoxinas detectadas

Tabela 5. Recuperação de micotoxinas nos sistemas de partição e seus coeficientes de variação

\begin{tabular}{cccccc}
\hline \multicolumn{9}{c}{ Recuperação \% $\left(\mathrm{CV}^{*}\right)$} \\
\hline Micotoxina & Experimento 1 & Experimento 2 & Experimento 3 & Experimento 4 & Experimento 5 \\
\hline AFA B $_{1}$ & $26,5(143)$ & $<$ LD & $53(9)$ & $<$ LD & $89(14)$ \\
AFA B $_{2}$ & $33,4(109)$ & $<$ LD & $70(1)$ & $<$ LD & $96(2)$ \\
OTA & $27(10)$ & $<$ LD & $40(1)$ & $<$ LD & $89(1)$ \\
ZEA & $\mathrm{Nd}$ & $97(4,5)$ & $80(14)$ & $<$ LD & $97(2)$ \\
DON & $\mathrm{Nd}$ & $7(1)$ & $60(1)$ & $<$ LD & $72(2)$ \\
\hline
\end{tabular}

*CV: Coeficiente de variação em \%; Nd: abaixo do limite de detecção. 
Tabela 6. Indicativos de mérito do método adaptado para determinação simultânea de micotoxinas em águas da cadeia produtiva do arroz

\begin{tabular}{|c|c|c|c|}
\hline $\begin{array}{c}\text { Concentração } \\
\text { inicial } \\
\left(\mathrm{ng} \mathrm{mL}^{-1}\right)\end{array}$ & $\begin{array}{c}\text { Concentração } \\
\text { recuperada } \\
\left(\mathrm{ng} \mathrm{mL} L^{-1}\right)\end{array}$ & $\begin{array}{l}\mathrm{CV} \\
(\%)\end{array}$ & $\begin{array}{c}\text { Recuperação } \\
(\%)\end{array}$ \\
\hline \multicolumn{4}{|c|}{$\mathrm{AFA} \mathrm{B}_{1}$} \\
\hline 9 & 8,0 & 4,1 & 89 \\
\hline 18 & 16 & 2,1 & 89 \\
\hline 27 & 24 & 4 & 89 \\
\hline \multicolumn{4}{|c|}{$\mathrm{AFA} \mathrm{B}_{2}$} \\
\hline 6 & 5,7 & 7,4 & 95 \\
\hline 12 & 11,5 & 4,6 & 96 \\
\hline 18 & 17,3 & 4,0 & 96 \\
\hline \multicolumn{4}{|c|}{ OTA } \\
\hline 15 & 13,2 & 12 & 88 \\
\hline 30 & 26,7 & 6,1 & 89 \\
\hline 60 & 53,4 & 11 & 89 \\
\hline \multicolumn{4}{|c|}{ ZEA } \\
\hline 70 & 67,9 & 8 & 97 \\
\hline 140 & 137,2 & 5 & 98 \\
\hline 210 & 205,8 & 12 & 98 \\
\hline \multicolumn{4}{|c|}{ DON } \\
\hline 65 & 47,5 & 4,0 & 73 \\
\hline 130 & 92,3 & 4,5 & 71 \\
\hline 260 & 187,2 & 4,8 & 72 \\
\hline
\end{tabular}

CV: Coeficiente de variação

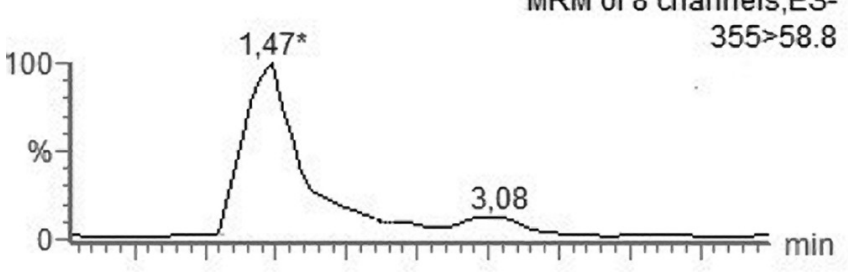

MRM of 8 channels,ES-

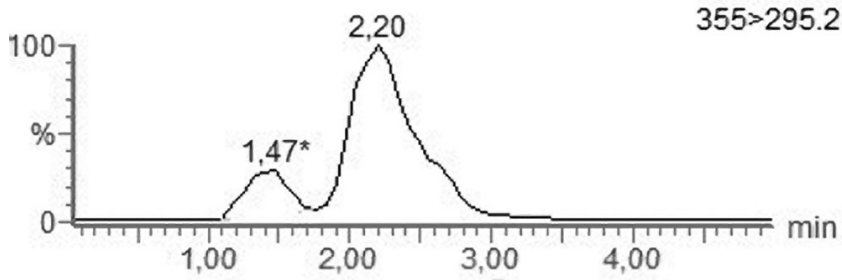

Figura 1. Cromatograma SRM obtido de amostra residuária do processo de parboilização contaminada com DON $\left(t_{R} 1,47\right)$

podem representar contaminação prévia por micota toxigênica no campo e/ou durante o armazenamento, que migrou para a água. Também indicam que efluentes industriais podem veicular toxinas naturais presentes no grão, além do risco de outros contaminantes já analisados rotineiramente.

\section{Migração de micotoxinas para água durante a parboilização}

A determinação do experimento laboratorial para avaliar a cinética de migração das micotoxinas para o grão foi motivada pela detecção delas nas amostras de água provenientes de empresas beneficiadoras de arroz. Além disso, Dors et al. ${ }^{10}$ e Coelho et al. ${ }^{14}$ já haviam demonstrado a migração das micotoxinas aqui estudadas para o interior do grão e, em algumas condições, os valores iniciais e finais sugeriam que uma parte da contaminação inicial poderia ter sido lixiviada para a água de encharcamento. Portanto, verificar o efeito do tempo de encharcamento e da contaminação inicial de grãos submetidos ao processo, através da determinação das micotoxinas na água, além de permitir avaliar a robustez do método validado, pode se constituir num subsídio interessante para a tomada de providências visando a manutenção da qualidade dos mananciais hídricos.

As condições de processo adotadas no experimento, com relação aos intervalos de encharcamento, observaram as faixas adotadas em empresas regionais, também empregadas em experimentos para estudos dos níveis de lixiviação de outros compostos químicos para o efluente de parboilização. ${ }^{10,12,13}$

Os resultados apresentados na Tabela 7 demonstram que o tempo de encharcamento é um fator importante na contaminação das águas residuais por micotoxinas, da mesma forma que para outros resíduos químicos. As micotoxinas ZEA, DON e OTA foram as que apresentaram os maiores percentuais de migração para água, 36, 30, 28\%, respectivamente.

Tabela 7. Migração de micotoxinas para água residuária de parboilização de grãos artificialmente contaminados

\begin{tabular}{cccccc}
\hline \multirow{2}{*}{ Micotoxinas } & \multirow{2}{*}{$\begin{array}{c}\text { Contaminação } \\
\text { N1/N2 } \\
\end{array}$} & \multicolumn{4}{c}{ Recuperação (\%) } \\
\cline { 3 - 6 } & $\left(\mu \mathrm{g} \mathrm{g}^{-1}\right)$ & $4 \mathrm{~h}$ & $6 \mathrm{~h}$ & $4 \mathrm{~h}$ & $6 \mathrm{~h}$ \\
\cline { 3 - 6 } & $0,17 / 0,4$ & 6 & 6 & 18 & 18 \\
AFA B $_{1}$ & $2,5 / 5$ & 12 & 12 & 24 & 30 \\
OTA & $10 / 20$ & $<\mathrm{LD}$ & 28 & 20 & 36 \\
ZEA & $12 / 16$ & 20 & 28 & 12 & 18 \\
DON & &
\end{tabular}

N1- Nível 1 de contaminação; N2- Nível 2 de contaminação; Nd- Não detectado

Dors et al. ${ }^{10}$ demonstraram que durante a parboilização ocorria um aumento do nível de contaminação de DON no endosperma, mas que parte da micotoxina ficou retida na casca do grão, cuja migração foi influenciada pelo tempo de encharcamento e nível de contaminação, o que foi consistente com os dados obtidos para a água. No presente estudo também foi demonstrada a ocorrência de redistribuição de solutos propiciada pelo processo de parboilização na etapa de encharcamento. Dors et al. ${ }^{10}$ atribuíram a permanência de DON na casca do grão à complexação com a sílica associada à celulose e lignina. No presente trabalho, esta afinidade de DON com a sílica ficou demonstrada pelo seu baixo Rf $(0,13)$ em CCDAE quando comparado ao das outras micotoxinas.

Estas observações sugerem que em caso de contaminação de arroz em casca com a micotoxina DON, a possibilidade da lixiviação para o interior do grão é maior do que para a água. Neste caso, a distribuição ocorre em função do tempo e da quantidade do contaminante. Quanto à contaminação observada nas amostras de água coletada em empresas, os níveis de contaminação da matéria-prima podem ter sido elevados, conforme visto nos resultados do experimento realizado.

Os baixos teores de migração das AFAs para a água estão consistentes com o estudo de Coelho et al. ${ }^{14}$ onde ficou demonstrada a migração destas toxinas para o interior do grão durante a etapa de encharcamento.

É importante salientar que, quando comparadas a outros solutos, tais como carboidratos, minerais e proteínas, ${ }^{15}$ a migração destas micotoxinas apresentou baixos percentuais em relação à contaminação 
inicial, o que já era esperado em função da pouca afinidade por solventes polares. No entanto, o risco de acúmulo nos corpos hídricos ficou demonstrado pelas amostras naturais e experimentais, podendo afetar a biota de mananciais e ocasionar danos na qualidade da água para as culturas, em processos industriais ou para consumo.

Considerando o perfil observado de migração de micotoxinas, seria importante avaliar suas interações com alguns solutos, visto que DON e AFAs podem ser adsorvidos por moléculas proteicas ou ter sua solubilidade alterada e migrar mais facilmente para o meio..$^{16,27,28}$

Dada a importância da exposição humana às micotoxinas para a saúde pública e as demonstrações de variáveis bióticas e abióticas que favorecem a produção de micotoxinas por espécies toxigênicas nas cadeias agropecuárias ${ }^{3,10,12,16,17}$ e os resultados do trabalho, ficou demonstrada a urgência da avaliação deste impacto nas águas residuárias antes de seu descarte pelas agroindústrias ou durante a drenagem de campos de cultivo. No caso das micotoxinas, esta necessidade é reforçada pelo fato delas serem termoestáveis e bioacumulativas, com consequente prejuízo ao meio ambiente, nem sempre facilmente associado à sua ocorrência nas águas dos mananciais hídricos.

\section{CONCLUSÃO}

A extração líquido-líquido de micotoxinas de amostras de águas da cadeia produtiva do arroz com clorofórmio, seguida por determinação em CCD ou CCDAE possibilitou a recuperação simultânea de AFA $B_{1}$, AFA $B_{2}$, OTA, ZEA e DON em níveis superiores a $70 \%$ e variabilidade inferior a $15 \%$, permitindo a proposição e validação de um método confiável, de fácil execução e com baixos teores de resíduos para descarte.

A detecção de micotoxinas em duas amostras de água residuária

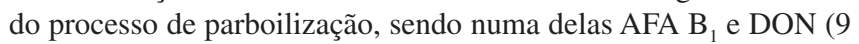
e $\left.110 \mathrm{ng} \mathrm{mL}^{-1}\right)$ e na outra DON (100 $\left.\mathrm{ng} \mathrm{mL}^{-1}\right)$, além de demonstrar a necessidade de cuidados com as matérias-primas empregadas e suas consequências no ambiente, indica a aplicabilidade do método validado.

O estudo de migração de micotoxinas de grãos artificialmente contaminados para a água residuária de parboilização reforçou a informação que o risco de contaminação existe, em especial para ZEA, OTA e DON.

\section{AGRADECIMENTOS}

$$
\text { Ao CNPq e ao IRGA. }
$$

\section{REFERÊNCIAS}

1. http://www.embrapa.br, acessada em Agosto 2010.

2. htpp://www.irga.rs.gov.br, acessada em Agosto 2010.

3. Nunes, I. L.; Magagni, G.; Bertolin, T. E.; Badiale-Furlong, E.; Boletim do SBCTA 2003, 23, 105.
4. Lima, C. A. P.; Orsi, R. B.; Dilkin, P.; Correa, B.; Ciên. Tecnol. Alimentos 2000, 20, 37.

5. Jaiaraman, P.; Kalyanasundaram, I.; Mycophatologia 1990, 110, 81.

6. Richard, J. J.; Int. J. Food Microbiology 2007, 119, 3.

7. Bennett, J. W.; Kich, M.; Clinical Microbiology Reviews 2003, 16, 497.

8. Yoshisawa, T.; Mycotoxin Analysis for Federative republic of Brazil, Training Course, Japão, 2001.

9. Ueno, Y.; Pure Appl.Chem. 1986, 58, 339.

10. Dors, G. C.; Pinto, L. A. A.; Badiale-Furlong, E.; Food Sci. Technol. Int. 2009, 42, 433.

11. Helen, C. S.; Hackbart, H. C. S.; Prietto, L.; Primel, E. G.; GardaBuffon, J.; Badiale-Furlong, E.; J. Braz. Chem. Soc. 2012, 23, 103.

12. Dors, G. C.; Bierhals, V. S.; Badiale-Furlong, E.; Ciên. Tecnol. Alimentos 2011, 31, 172.

13. Brasil; Ministério da Saúde, Agência Nacional de Vigilância Sanitária; Resolução - RDC n ${ }^{\circ}$ 7; Diário Oficial da República Federativa do Brasil, Brasília, DF, 18/02/2011.

14. Coelho, C. S.; Badiale-Furlong, E.; Almeida, T. L; Brazilian Journal Food Technology 1999, 2, 43.

15. Queiroz, M. I.; Koetz, P. R.; Revista Brasileira Agrociência 1997, 3 , 139.

16. Garda-Buffon, J.; Macedo, R. M.; Badiale-Furlong, E.; Ciên. Tecnol. Alimentos 2004, 24, 657.

17. Trindade, E.; Furlong, E. B.; Noll, I. B.; Boletim do SBCTA 2001, 9, 2.

18. Soares, L. M. V.; Rodriguez-Amaya, D.; J. Assoc. Off. Anal.Chem. 1989, $72,22$.

19. Colins, C.; Scientia Chromatographica 2010, 2,5

20. A.O.A.C International; Official Methods of International, $16^{\text {th }}$ ed. $4^{\text {th }}$ ver. 1998 (Software Adobe and E-Doc/C.J.S).

21. Baraj, E.; Garda-Buffon, J.; Badiale-Furlong, E.; Braz. Arch. Biol. Technol. 2010, 53, 505

22. Lin, L.; Zhang, J.; Wang, P.; Wang, Y.; Chen, J.; J. Chromatogr., A 1998, 815,3 .

23. Ribani, M.; Bottoli, C. B. G.; Collins, C. H.; Jardim, I. C. S. F.; Melo, L.; Quim. Nova 2004, 27, 771.

24. European Communities; Commission Regulation 401/2006; Off. J. Eur. Commun. 2006, 70, 12

25. INMETRO - Instituto Nacional de Metrologia, Normalização e Qualidade Industrial; DOQ-CGCRE-008, Orientações sobre validação de métodos de ensaios químicos, INMETRO: Rio de Janeiro, 2007.

26. Scott, P. M.; J. AOAC Int. 1982, 65, 876.

27. Scott, P. M.; Kanhere, S. R.; Daley, E. F.; Faber, J. M.; Food Addit. Contam. 1993, 10, 381.

28. Scott, P. M.; Kanhere, S. R.; Food Addit. Contam. 1995, 12, 591.

29. Kotal, F.; Holadová, K.; Hajsçová, J.; Poustka, J.; Radová, Z. ; J. Chromatogr., A 1999, 830, 219.

30. Dors, J. C.; Tese de Doutorado, Universidade Federal do Rio Grande, Brasil, 2010.

31. Jardim, I. C. S. F.; Scientia Chromagraphica 2010, 2, 13.

32. Nováková, L.; Vlcková, H.; Anal. Chim. Acta 2009, 656, 8. 